\title{
Influence of solifenacin on the improvement of storage symptoms in the early period after photoselective vaporization of the prostate
}

\author{
Su Jin $\operatorname{Kim}^{1} \oplus$, Woong Jin $\mathrm{Bae}^{2,3} \mathbb{1}$, Sae Woong $\mathrm{Kim}^{2,3} \mathbb{1}$ \\ 'Department of Urology, Yonsei University Wonju College of Medicine, Wonju, ${ }^{2}$ Department of Urology, The Catholic University of Korea, Seoul St. Mary's Hospital, College \\ of Medicine, The Catholic University of Korea, Seoul, ${ }^{3}$ Catholic Integrative Medicine Research Institute, The Catholic University of Korea, Seoul, Korea
}

Purpose: We studied the effect of solifenacin on reducing storage symptoms after photoselective vaporization of the prostate (PVP). Materials and Methods: This study included patients with persistent storage symptoms of urgency and frequency in a 3-day voiding diary, International Prostate Symptom Score (IPSS) storage subscore (IPSS-s) $\geq 5$, overactive bladder symptom score (OABSS) $\geq 5$, and OABSS for question $3 \geq 4$ at 5 days after urethral catheter removal. The patients were randomly assigned to receive once-daily solifenacin $5 \mathrm{mg}$ or placebo for 4 weeks. Evaluation of the 3-day voiding diary, IPSS, and OABSS was performed at 2 and 4 weeks after treatment.

Results: At 2 and 4 weeks after treatment, the urgency and frequency in the 3-day voiding diary, IPSS, IPSS-s, and OABSS were decreased in the solifenacin group. Although the OABSS of the solifenacin group was not significantly different from that of the placebo group, the OABSS of the placebo group increased at 4 weeks compared with that at 2 weeks after treatment. The Benefit, Satisfaction, and Willingness to continue questionnaire showed no significant difference in patient satisfaction between the groups. Although the solifenacin group showed increased post-void residual volume compared with the placebo group, there was no statistically significant difference.

Conclusions: Storage symptoms measured using OABSS tended to decrease after medication with solifenacin in the early period after PVP. Therefore, we suggest that anticholinergics have a potential role in improving storage symptoms after PVP.

Keywords: Laser therapy; Lower urinary tract symptoms; Muscarinic antagonists; Postoperative period; Prostatic hyperplasia

This is an Open Access article distributed under the terms of the Creative Commons Attribution Non-Commercial License (http://creativecommons.org/licenses/by-nc/4.0) which permits unrestricted non-commercial use, distribution, and reproduction in any medium, provided the original work is properly cited.

\section{INTRODUCTION}

Benign prostatic hyperplasia (BPH) is a common voiding dysfunction in elderly males. Surgical treatment is considered for patients with complications associated with $\mathrm{BPH}$ and persistent voiding problems despite treatment with
BPH medicine [1]. For these patients, surgical treatment such as transurethral resection of the prostate (TURP) and transurethral laser surgery with holmium:yttrium-aluminum-garnet and GreenLight laser have been performed. TURP is a gold standard surgical method with good efficacy and a low adverse event rate, and use of transurethral laser surgery

Received: 9 February, 2019 - Accepted: 6 August, 2019

Corresponding Author: Sae Woong Kim (iD https://orcid.org/0000-0002-9127-9400

Department of Urology, The Catholic University of Korea, Seoul St. Mary's Hospital, College of Medicine, The Catholic University of Korea, 222 Banpo-daero, Seocho-gu, Seoul 06591, Korea

TEL: +82-2-2258-6226, FAX: +82-2-599-7839, E-mail: ksw1227@catholic.ac.kr 
has been increasing [2]. However, some patients experience persistent or newly developed lower urinary tract symptoms (LUTSs) after BPH surgery, with previous studies reporting that $5 \%$ to $35 \%$ of patients complain of LUTSs after TURP [3]. The various voiding and storage LUTSs observed after $\mathrm{BPH}$ surgery are considered the result of chronic bladder outlet obstruction (BOO).

After surgery for relieving BOO, about $20 \%$ to $40 \%$ of patients show unresolved detrusor overactivity (DO) as well as storage symptoms such as frequency and urgency [4,5]. As a result, about one-third of patients need additional treatment to improve frequency and urgency after BPH surgery. The problems of persistent frequency and urgency even after the relief of $\mathrm{BOO}$ may originate from the bladder without being related to $\mathrm{BOO}$ and $\mathrm{BPH}$ or bladder changes induced by chronic BOO [6,7]. However, there is no useful method to predict which patient will experience persistent storage symptoms after BPH surgery. Therefore, proper treatment to improve postoperatively persistent storage symptoms is important to increase the patients' quality of life (QoL) [8,9]. Thus, in this study, we evaluated the efficacy and adverse events of the antimuscarinic agent solifenacin in patients with persistent storage symptoms after photoselective vaporization of the prostate (PVP).

\section{MATERIALS AND METHODS}

\section{Patients}

This study enrolled males aged $>50$ years who underwent PVP surgery for BPH, with persistent storage symptoms of urgency and frequency ( $>3$ urgency episodes and $>8$ micturition events every 24 hours recorded in a 3-day voiding diary), International Prostate Symptom Score (IPSS) storage subscore $\geq 5$, overactive bladder symptom score (OABSS) $\geq 5$, and OABSS for question $3 \geq 4$ at 5 days after urethral catheter removal. On the other hand, patients with clinically severe BOO; those with post-void residual (PVR) volume $\geq 100 \mathrm{~mL}$; patients with prostate, bladder, or other pelvic cancer, those with neurogenic conditions including multiple sclerosis, stroke, dementia, spinal cord injury, or Parkinson's disease; those with gastrointestinal obstructive diseases, narrow angle glaucoma, liver disease, renal disease, diabetic neuropathy, or urinary tract infection; and males taking anticholinergics within 14 days of the trial were excluded. This study was conducted in accordance with the Declaration of Helsinki and was approved by the Institutional Review Board of The Catholic University of Korea (approval number: KC12MSSV0610). All patients provided written informed consent.

\section{Study design}

This was a randomized, double-blinded, placebo-controlled study. Initial screening assessment was conducted in patients who were planned to undergo PVP operation for the treatment of $\mathrm{BPH}$. The basic screening tests included medical and surgical history taking, collecting details of current pharmacological therapy, blood pressure measurement, and laboratory tests. All eligible patients were randomized into the solifenacin succinate group (study group) or the placebo group (control group) at a 1:1 ratio. Randomization was performed by using a computerized randomization table, and the order was prepared by the statistical analysis team of a contract research organization (CMIC, Bucheon, Korea). The patients were assigned to groups by order from the smallest number at the center. For balanced randomization in both groups, a stratified randomized complete block design was used, with the surgical method (PVP) as a stratifying factor.

PVP was performed by a single urologist (SW Kim), and all procedures were carried out with the patients under general or spinal anesthesia. A continuous running irrigation system 22-Fr resectoscope and a laser fiber were used. The 120-W high-performance system GreenLight laser (Laserscope, GreenLight; American Medical Systems, Minnetonka, MN, USA) was used, and all prostate tissues causing obstruction were removed until a fine surgical cavity was formed.

An 18-Fr urethral catheter was placed after the operation, and irrigation with saline solution was started in the operating room. Irrigation was stopped after the patients recovered from anesthesia and if the urine was clear. After PVP, urethral catheters were removed from all patients on the next day after the operation, and patients were dis-

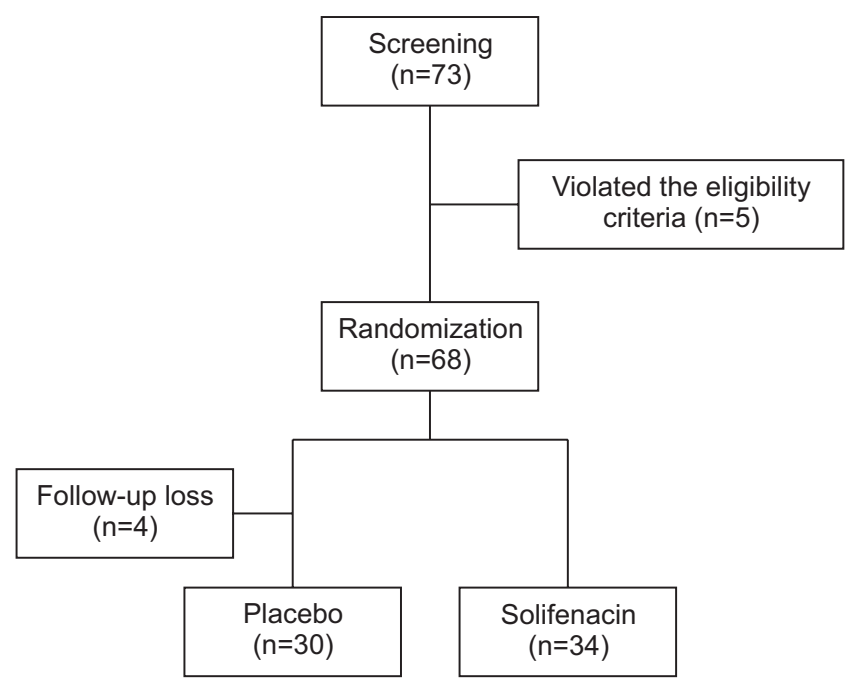

Fig. 1. Study flowchart. 
charged from the hospital without urethral catheters. The patients visited the outpatient clinic at 5 days after urethral catheter removal, and storage symptoms were assessed using the records in the 3-day voiding diary, IPSS storage subscore, and OABSS. By using random number tables, the patients were randomly assigned to receive once-daily solifenacin 5 mg or placebo for 4 weeks (Fig. 1).

\section{Efficacy and safety assessment}

Efficacy was assessed on the basis of the change of urgency per day from the 3-day voiding diary at 2 and 4 weeks after surgery. In addition, the change of frequency per day in the 3-day voiding diary was analyzed at 2 and 4 weeks after surgery. The changes of IPSS (total, storage subscore, voiding subscore, and QoL score) and OABSS were compared between the placebo and solifenacin groups at 2 and 4 weeks after PVP. Patient satisfaction was compared using the Benefit, Satisfaction, and Willingness to continue (BSW) questionnaire. The maximum flow rate (Qmax) and PVR volume were evaluated at 2 and 4 weeks after surgery. All adverse events were collected.

\section{Statistical analysis}

Statistical analyses were performed using SAS version 9.2 statistical software (SAS Institute, Cary, NC, USA). All statistical assessments were 2-sided and considered significant at $\mathrm{p}<0.05$. In this study, we referred to the results of a previous study [10] that administered solifenacin $5 \mathrm{mg}$ and placebo to patients who showed $\mathrm{OAB}$ even after the removal of the catheter following PVP. In the previous study, the difference in the mean changes of urgency episodes per 24 hours from the baseline to the endpoint (2 weeks) between the treatment $(-2.4 \pm 1.3)$ and placebo $(-1.3 \pm 0.8)$ groups was 1.1. The difference in the mean changes from baseline to 2 weeks between the groups was conservatively assumed to have been 1.1, and the standard deviations for both groups were assumed to have been 1.3. The minimum number of subjects in each group was set at 31 to achieve $90.6 \%$ statistical power to detect the difference using the t-test, with a 2-sided significance level of $5 \%$. Considering a $10 \%$ dropout rate, 70 patients ( 35 patients in each group) were planned to be enrolled.

The efficacy evaluation in this study was mainly performed in the modified intention-to-treat (MITT) set of subjects who were treated with the investigational product and who had at least 1 primary efficacy evaluation, and additionally in the per-protocol set. The final evaluation for interpreting the results was based on the results from the MITT set. A safety analysis was performed using the intention-to-treat set that included patients who were randomized and followed-up at least once.

Statistical analyses using analysis of covariance (ANCOVA) were performed to calibrate the significant differences of baseline IPSS between the solifenacin and placebo groups. The mean change in daily (24-hour) urinary urgency was based on the voiding diary completed for 3 consecutive days before the visit at 2 weeks, compared with the baseline. The mean changes in the following items at 2 and 4 weeks compared with the baseline values were analyzed in the solifenacin and placebo groups: total score, storage subscale score, voiding subscale score, and QoL score in the IPSS questionnaire; OABSS total score; and daily (24-hour) number of micturition events based on the voiding diary completed for 3 consecutive days before the visits.

\section{RESULTS}

From December 2012 to October 2013, 64 patients were assigned to either the placebo group $(\mathrm{n}=30)$ or the solifenacin group $(n=34)$ according to the inclusion criteria. The baseline

Table 1. Baseline characteristics at 1 week after surgery

\begin{tabular}{lccc}
\hline \multicolumn{1}{c}{ Characteristic } & Placebo & Solifenacin & p-value \\
\hline Age $(y)$ & $69.76 \pm 8.31(56.0-88.0)$ & $71.09 \pm 6.79(59.0-83.0)$ & 0.47 \\
Mean number of micturition events per 24 hours & $11.31 \pm 1.77(8.67-18.33)$ & $11.18 \pm 1.55(8.33-14.33)$ & 0.96 \\
Mean number of urgency episodes per 24 hours & $7.45 \pm 2.70(4.0-14.0)$ & $7.60 \pm 2.81(3.67-14.0)$ & 0.79 \\
OABSS & $8.65 \pm 1.92(6.0-14.0)$ & $8.71 \pm 1.78(6.0-13.0)$ & 0.75 \\
IPSS total score & $16.74 \pm 5.70(9.0-30.0)$ & $19.68 \pm 5.35(11.0-30.0)$ & $0.02^{*}$ \\
IPSS storage subscore & $7.82 \pm 1.73(5.0-13.0)$ & $9.24 \pm 2.05(5.0-14.0)$ & $0.00^{*}$ \\
IPSS voiding subscore & $8.91 \pm 4.78(3.0-20.0)$ & $10.44 \pm 4.38(3.0-19.0)$ & 0.11 \\
IPSS QoL score & $3.29 \pm 0.97(1.0-5.0)$ & $3.56 \pm 0.70(3.0-5.0)$ & 0.18 \\
\hline
\end{tabular}

Values are presented as mean \pm standard deviation (range). Analysis was performed in randomized patients $(n=68)$.

OABSS, overactive bladder symptom score; IPSS, International Prostate Symptom Score; QoL, quality of life.

${ }^{*} \mathrm{p}<0.05$ compared with the placebo group. 
characteristics of the placebo and solifenacin groups are shown in Table 1 . The mean age was similar in the placebo group (69.76 \pm 8.31 years) and the solifenacin group (71.09 \pm 6.79 years). From the 3-day voiding diary, the mean number of micturition events per 24 hours was similar in the placebo $(11.31 \pm 1.77)$ and solifenacin $(11.18 \pm 1.55)$ groups. The mean number of urgency episodes per 24 hours was also similar in the placebo (7.45 \pm 2.70$)$ and solifenacin $(7.60 \pm 2.81)$ groups. The IPSS voiding subscore and the IPSS QoL score were similar in the placebo and solifenacin groups. The total IPSS and the IPSS storage subscore (19.68 \pm 5.35 and $9.24 \pm 2.05$, respectively) of the solifenacin group were significantly higher than those of the placebo group $(16.74 \pm 5.70$ and $7.82 \pm 1.73$, respectively) $(p<0.05)$. However, the mean OABSS was similar in the placebo (8.65 \pm 1.92$)$ and solifenacin $(8.71 \pm 1.78)$ groups.

\section{Changes of urgency and frequency}

In the solifenacin group, urgency was decreased at 2 and 4 weeks after surgery compared with the baseline (Table 2). However, there was no significant difference compared with the placebo group at 2 and 4 weeks after surgery. Similarly, frequency was decreased at 2 and 4 weeks after surgery compared with baseline in the solifenacin group, but there was no significant difference compared with the placebo group.

\section{Changes of subjective symptoms}

The IPSS (total score, voiding subscore, and storage subscore) of the solifenacin group at 2 and 4 weeks after surgery decreased compared with the baseline (Fig. 2). However, there was no significant difference compared with the placebo group. The IPSS QoL score of the solifenacin group was improved after surgery compared with baseline, but there was no significant difference compared with the placebo group.

The OABSS of the solifenacin group decreased at 2 and 4 weeks after surgery compared with the baseline; however, there was no significant difference compared with the placebo group. Although there was no significant difference, the OABSS of the placebo group at 4 weeks increased compared with that at 2 weeks after surgery. However, the OABSS continuously decreased until 4 weeks after surgery in the solifenacin group.

Table 2. Changes of urgency and frequency in the placebo and solifenacin groups

\begin{tabular}{cccc}
\hline Variable & $\begin{array}{c}\text { Placebo } \\
(\mathrm{n}=30)\end{array}$ & $\begin{array}{c}\text { Solifenacin } \\
(\mathrm{n}=36)\end{array}$ & $\mathrm{p}$-value \\
\hline Urgency & & & \\
Baseline & $7.60 \pm 2.81$ & $7.60 \pm 2.82$ & 0.79 \\
2 weeks & $3.79 \pm 3.88$ & $3.82 \pm 3.51$ & 0.97 \\
4 weeks & $3.14 \pm 3.92$ & $3.65 \pm 3.32$ & 0.53 \\
Frequency & & & \\
Baseline & $11.46 \pm 1.81$ & $11.18 \pm 1.55$ & 0.96 \\
2 weeks & $9.20 \pm 1.86$ & $9.19 \pm 2.18$ & 0.81 \\
4 weeks & $9.02 \pm 2.23$ & $8.77 \pm 1.77$ & 0.72 \\
\hline
\end{tabular}

Values are presented as mean \pm standard deviation. Analysis was performed in patients who completed primary efficacy assessment $(n=64)$. Baseline means 1 week after surgery.
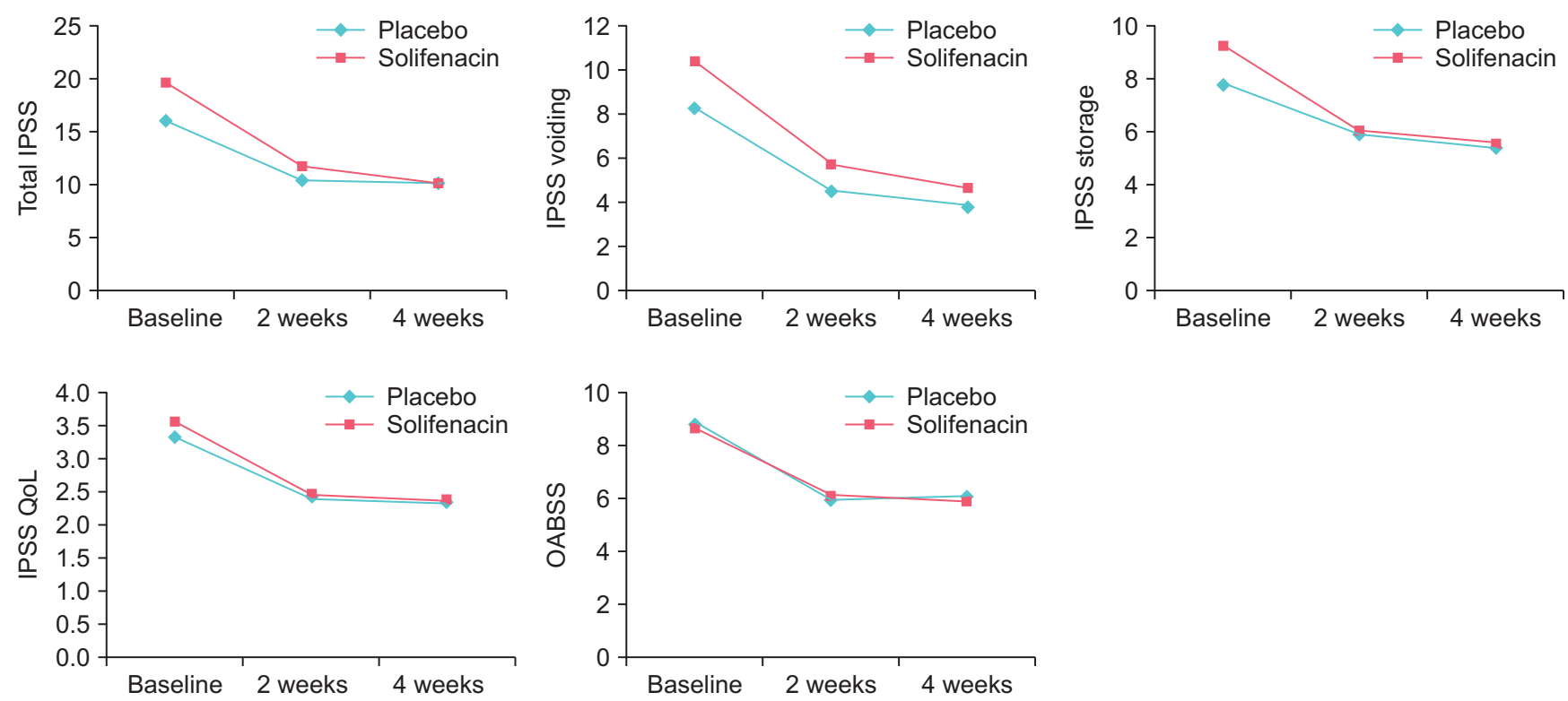

Fig. 2. Changes of subjective symptoms after photoselective vaporization of the prostate. IPSS, International Prostate Symptom Score; QoL, quality of life; OABSS, overactive bladder symptom score. 
Table 3. Comparison of the patient satisfaction between the placebo and solifenacin groups

\begin{tabular}{|c|c|c|c|c|}
\hline \multirow{2}{*}{ Variable } & \multicolumn{2}{|c|}{ Placebo $(n=30)$} & \multicolumn{2}{|c|}{ Solifenacin $(n=34)^{a}$} \\
\hline & 2 weeks & 4 weeks & 2 weeks & 4 weeks \\
\hline \multicolumn{5}{|c|}{ Have you had any benefit from your treatment? } \\
\hline Much benefit & $16(53.3)$ & $17(56.7)$ & $17(50.0)$ & $19(55.9)$ \\
\hline Little benefit & $11(36.7)$ & $11(36.7)$ & $16(47.1)$ & $11(32.4)$ \\
\hline No & $3(10.0)$ & $2(6.7)$ & $1(2.9)$ & $4(11.8)$ \\
\hline \multicolumn{5}{|c|}{ Taking all things into account, are you satisfied with your treatment? } \\
\hline Very satisfied & $11(36.7)$ & $16(53.3)$ & $13(38.2)$ & $13(38.2)$ \\
\hline A little satisfied & $12(40.0)$ & $8(26.7)$ & $18(52.9)$ & $14(41.2)$ \\
\hline A little dissatisfied & $6(20.0)$ & $6(20.0)$ & $3(8.8)$ & $7(20.6)$ \\
\hline Very dissatisfied & $1(3.3)$ & $0(0.0)$ & $0(0.0)$ & $0(0.0)$ \\
\hline \multicolumn{5}{|c|}{ Would you be willing to continue treatment with this medication? } \\
\hline Very willing & $6(20.0)$ & $8(26.7)$ & $11(32.4)$ & $10(29.4)$ \\
\hline A little bit willing & $13(43.3)$ & $14(46.7)$ & $13(38.2)$ & $10(29.4)$ \\
\hline A little bit unwilling & $11(36.7)$ & $8(26.7)$ & $9(26.5)$ & $13(38.2)$ \\
\hline Very unwilling & $0(0.0)$ & $0(0.0)$ & $1(2.9)$ & $0(0.0)$ \\
\hline
\end{tabular}

Values are presented as number (\%).

${ }^{\mathrm{a}}$ :There was no significant difference between the placebo and solifenacin groups.

\section{Patient satisfaction}

The results of the BSW questionnaire in the solifenacin group was not significantly different from those in the placebo group at 2 and 4 weeks after surgery (Table 3).

\section{Adverse events}

The Qmax of the solifenacin group increased at 2 and 4 weeks after surgery compared with the baseline value; however, there was no significant difference compared with the placebo group (Table 4). The PVR volume of the solifenacin group (64.63 $\pm 56.41 \mathrm{~mL})$ showed a higher increase at 4 weeks than that of the placebo group $(40.29 \pm 34.03 \mathrm{~mL})$, although there was no significant difference.

There were no significant adverse events in both the placebo and solifenacin groups. In the placebo group, hematuria (2/30), dysuria (1/30), and insomnia (1/30) were noted. Abdominal discomfort (1/34), constipation (1/34), and urticaria (1/34) were observed in the solifenacin group.

\section{DISCUSSION}

Approximately one-third of patients experience persistent DO or storage symptoms after BPH surgery, and these symptoms reduce the patients' satisfaction after surgery as well as decrease the QoL despite the disappearance of obstructive symptoms. Therefore, it is important to reduce persistent storage symptoms after BPH surgery. In the present study, we performed a randomized, double-blinded, placebocontrolled investigation of the efficacy and adverse events of solifenacin $5 \mathrm{mg}$ in patients undergoing PVP surgery for
Table 4. Changes of Qmax and PVR volume between the placebo and solifenacin groups

\begin{tabular}{ccc}
\hline \multicolumn{1}{c}{ Variable } & Placebo & Solifenacin $^{\mathrm{a}}$ \\
\hline Qmax $(\mathrm{mL} / \mathrm{s})$ & & \\
Baseline & $10.96 \pm 7.87(1.40-33.0)$ & $8.96 \pm 5.69(1.70-31.90)$ \\
2 weeks & $15.74 \pm 9.05(15.74-9.05)$ & $12.72 \pm 7.03(2.50-32.70)$ \\
4 weeks & $17.60 \pm 9.23(4.60-44.90)$ & $16.30 \pm 8.25(3.40-33.40)$ \\
PVR volume $(\mathrm{mL})$ & \\
Baseline & $39.03 \pm 25.33(9.0-92.0)$ & $44.91 \pm 32.49(7.0-99.0)$ \\
2 weeks & $39.87 \pm 35.46(0.0-151.0)$ & $61.00 \pm 61.32(0.0-277.0)$ \\
4 weeks & $40.29 \pm 34.03(7.0-134.0)$ & $64.63 \pm 56.41(6.0-207.0)$ \\
\hline
\end{tabular}

Values are presented as mean \pm standard deviation (range).

Qmax, maximum flow rate; PVR, post-void residual.

${ }^{a}:$ There was no significant difference between the placebo and solifenacin groups. Baseline means 1 week after surgery.

the treatment of $\mathrm{BPH}$. Although there were no significant differences compared with the placebo group, urgency and frequency were decreased in the solifenacin group at the 4-week follow-up compared with the baseline. In addition, subjective symptoms assessed using the IPSS and OABSS also improved at 4 weeks after surgery compared with the baseline. Moreover, the important finding of this study is that the OABSS of the solifenacin group showed a continuously decreasing tendency until 4 weeks of follow-up. In contrast, the OABSS of the placebo group was higher at 4 weeks than at 2 weeks after surgery. Further, there were no significant adverse events after medication with solifenacin.

In the present study, the baseline IPSS storage subscore of the solifenacin group was significantly different from that of the placebo group, and the significant difference of 
the total IPSS between the 2 groups was due to the difference in the IPSS storage subscore. Therefore, statistical analysis using ANCOVA was performed in the present study to exclude the influence of the baseline difference between the 2 groups. The results of this study are reliable because the comparison was performed after statistical modification.

Several investigators have attempted to find the risk factors associated with persistent urgency and frequency after the relief of $\mathrm{BOO}$ through $\mathrm{BPH}$ surgery, because persistent storage symptoms reduce the patients' satisfaction after surgery and additional treatment is necessary to control unresolved symptoms.

Most of the previous studies considered preoperative DO as one of the risk factors for persistent DO after surgery, and there have been various reports about the influence of preoperative DO on the surgical outcomes. Tanaka et al. [11] analyzed the influence of the degree of BOO, DO, and detrusor underactivity on preoperative urodynamic evaluations, and noted that preoperative DO did not influence the outcome of TURP. However, they also observed that the treatment efficacy was lower in patients with DO but not BOO on urodynamics than those with a severe degree of BOO.

Moreover, several studies analyzed the influence of preoperative DO that was subdivided according to the characteristics on urodynamics on postoperative urgency and frequency. A previous study found that continuous DO, compared with a single episode of $\mathrm{DO}$, on urodynamics was associated with persistent DO [8]. Other studies observed that preoperative terminal DO was associated with poorer outcome and earlier appearance of DO, and that a higher amplitude of $>40 \mathrm{~cm} \mathrm{H}_{2} \mathrm{O}$ was strongly associated with persistent DO after TURP [9,12].

Further, more severe storage symptoms are associated with the possibility of the existence of preoperative DO because previous studies reported that the presence of $\mathrm{DO}$ is related to more significantly impaired symptoms [13,14]. However, we could not conclude that the solifenacin group had a higher likelihood of having preoperative DO because there is no established relationship between presence of DO and the IPSS storage subscore.

From the various surgical treatment methods, we chose PVP because this procedure is considered to be more associated with postoperative storage symptoms than other procedures $[15,16]$. A higher rate of storage or irritative symptoms may occur due to dysuria after PVP [17]. In the urodynamic study after PVP, a previous report showed that urge urinary incontinence occurred in half of the patients until the 9-month follow-up after PVP and disappeared at 12 months
[18]. Moreover, Cho et al. [19] reported that $24.4 \%$ of their patients started anticholinergics at a mean of 1.6 months after PVP and maintained the medication for a mean of 6.6 months. Different from the previous investigations, the patients in the present study were followed-up after a short period. Most of the previous studies started to evaluate the patients' symptoms at 1 month after surgery, and decided whether or not to start additional therapy. However, we started to evaluate the patients' symptoms at 7 days after catheter removal and followed-up the patients at 2 and 4 weeks after PVP. Therefore, our results are difficult to compare with those of previous studies. However, our study provides information about the short-term changes of storage symptoms. Most of the improvements of the IPSS storage subscore and the OABSS compared with the baseline values occurred until 2 weeks in both the placebo and solifenacin groups. Thereafter, the changes in the IPSS storage subscore and the OABSS of the placebo and solifenacin groups were minimal from 2 to 4 weeks. From these results, we assumed that the improvement of storage symptoms resulted from the disappearance of dysuria associated with PVP until 2 weeks, and this may be the reason why the solifenacin group did not show a significant improvement compared with the placebo group.

The solifenacin group showed lower OABSS at 4 weeks than that at 2 weeks after PVP. On the contrary, the placebo group showed higher OABSS at 4 weeks than that at 2 weeks after PVP. These changes may reflect the real persistent storage symptoms in the placebo group after the resolution of dysuria induced by PVP. Moreover, these results are in line with those of previous long-term follow-up studies about storage symptoms after PVP. Therefore, solifenacin may help improve persistent storage symptoms after PVP.

Although the findings of the present study are consistent with those of previous reports, there was no significant difference between the solifenacin and placebo groups. This limitation may be associated with the short-term follow-up period in this study. In addition, the differences in the preoperative storage symptoms between the solifenacin and placebo groups may have influenced the results, even though we analyzed the data after adjustment. Moreover, the discrepancy in the IPSS storage subscore and the OABSS at 4 weeks could decrease the reliability of the results. However, we believe that storage symptoms evaluated using OABSS can reflect the patients' real symptoms because the OABSS and the IPSS storage subscore are not always correlated with each other [20]. 


\section{CONCLUSIONS}

Solifenacin reduced the storage symptoms of patients after PVP, although there were no significant differences compared with the storage symptoms of the placebo group. However, storage symptoms measured using the OABSS tended to decrease at 4 weeks after medication with solifenacin. Therefore, anticholinergics may contribute to the improvement of storage symptoms in the early period after PVP.

\section{CONFLICTS OF INTEREST}

The authors have nothing to disclose.

\section{ACKNOWLEDGMENTS}

The study was funded by Astellas Korea Co. Ltd., and designed and analyzed by Astellas in collaboration with the authors. Editorial assistance was funded by Astellas Pharma Inc.

\section{AUTHORS' CONTRIBUTIONS}

Research conception \& design: Sae Woong Kim and Su Jin Kim. Data analysis and interpretation: Woong Jin Bae. Drafting of the manuscript: Su Jin Kim. Approval of the final manuscript: all authors.

\section{REFERENCES}

1. Yeo JK, Choi H, Bae JH, Kim JH, Yang SO, Oh CY, et al. Korean clinical practice guideline for benign prostatic hyperplasia. Investig Clin Urol 2016;57:30-44.

2. Mandeville J, Mourtzinos A. Surgical management of male voiding dysfunction. Surg Clin North Am 2016;96:491-501.

3. Nitti VW, Kim Y, Combs AJ. Voiding dysfunction following transurethral resection of the prostate: symptoms and urodynamic findings. J Urol 1997;157:600-3.

4. Abrams PH, Farrar DJ, Turner-Warwick RT, Whiteside CG, Feneley RC. The results of prostatectomy: a symptomatic and urodynamic analysis of 152 patients. J Urol 1979;121:640-2.

5. Gormley EA, Griffiths DJ, McCracken PN, Harrison GM, McPhee MS. Effect of transurethral resection of the prostate on detrusor instability and urge incontinence in elderly males. Neurourol Urodyn 1993;12:445-53.

6. Hyman MJ, Groutz A, Blaivas JG. Detrusor instability in men: correlation of lower urinary tract symptoms with urodynamic findings. J Urol 2001;166:550-2.
7. Housami F, Abrams P. Persistent detrusor overactivity after transurethral resection of the prostate. Curr Urol Rep 2008;9:284-90.

8. Kageyama S, Watanabe T, Kurita Y, Ushiyama T, Suzuki K, Fujita K. Can persisting detrusor hyperreflexia be predicted after transurethral prostatectomy for benign prostatic hypertrophy? Neurourol Urodyn 2000;19:233-40.

9. Zhao YR, Liu WZ, Guralnick M, Niu WJ, Wang Y, Sun G, et al. Predictors of short-term overactive bladder symptom improvement after transurethral resection of prostate in men with benign prostatic obstruction. Int J Urol 2014;21:1035-40.

10. YI Q, Gong M, Hu W, Tian B, Zhu F, Wang T, et al. Efficacy of solifenacin in the treatment of overactive bladder syndrome after transurethral resection of the prostate. Chin J Urol 2011;32:415-8.

11. Tanaka Y, Masumori N, Itoh N, Furuya S, Ogura H, Tsukamoto T. Is the short-term outcome of transurethral resection of the prostate affected by preoperative degree of bladder outlet obstruction, status of detrusor contractility or detrusor overactivity? Int J Urol 2006;13:1398-404.

12. Antunes AA, Iscaife A, Reis ST, Albertini A, Nunes MA, Lucon $\mathrm{AM}$, et al. Can we predict which patients will experience resolution of detrusor overactivity after transurethral resection of the prostate? J Urol 2015;193:2028-32.

13. Jeong SJ, Lee SC, Jeong CW, Hong SK, Byun SS, Lee SE. Clinical and urodynamic differences among women with overactive bladder according to the presence of detrusor overactivity. Int Urogynecol J 2013;24:255-61.

14. Giarenis I, Mastoroudes H, Srikrishna S, Robinson D, Cardozo L. Is there a difference between women with or without detrusor overactivity complaining of symptoms of overactive bladder? BJU Int 2013;112:501-7.

15. Hamann MF, Naumann CM, Seif C, van der Horst C, Jünemann KP, Braun PM. Functional outcome following photoselective vaporisation of the prostate (PVP): urodynamic findings within 12 months follow-up. Eur Urol 2008;54:902-7.

16. Matoka DJ, Averch TD. Predictability of irritative voiding symptoms following photoselective laser vaporization of the prostate. Can J Urol 2007;14:3710-4.

17. Al-Ansari A, Younes N, Sampige VP, Al-Rumaihi K, Ghafouri A, Gul T, et al. GreenLight HPS 120-W laser vaporization versus transurethral resection of the prostate for treatment of benign prostatic hyperplasia: a randomized clinical trial with midterm follow-up. Eur Urol 2010;58:349-55.

18. Pereira-Correia JA, de Moraes Sousa KD, Santos JB, de Morais Perpétuo D, Lopes-da-Silva LF, Krambeck RL, et al. GreenLight HPS $^{\text {ma }} 120-\mathrm{W}$ laser vaporization vs transurethral resection of the prostate $(<60 \mathrm{~mL})$ : a 2-year randomized double-blind prospective urodynamic investigation. BJU Int 2012;110:1184-9. 
19. Cho MC, Ha SB, Oh SJ, Kim SW, Paick JS. Change in storage symptoms following laser prostatectomy: comparison between photoselective vaporization of the prostate (PVP) and holmium laser enucleation of the prostate (HoLEP). World J Urol 2015;33:1173-80.
20. Shim JS, Kim JH, Choi H, Park JY, Bae JH. Diagnostic tool for assessing overactive bladder symptoms: could the international prostate symptom storage subscore replace the overactive bladder symptom score? Int Neurourol J 2016;20:209-13. 